\title{
Towards supramolecular nanostructured materials: Control of the self-assembly of ionic bent-core amphiphiles
}

Received 00th January 20xx, Accepted 00th January 20xx DOI: $10.1039 / x 0 \times x 00000 x$

\author{
Martín Castillo-Vallés, ${ }^{a}$ Miguel Cano, ${ }^{\mathrm{b}}$ Ana Bermejo-Sanz, ${ }^{\mathrm{c}}$ Nélida Gimeno, ${ }^{\mathrm{d}}$ M. Blanca Ros ${ }^{\mathrm{a*}}$
}

Control of the bottom-up self-assembly of ionic bent-core dendrimers has been systematically studied by molecular design. This process gave rise to architectures with diverse packing, shapes and dimensions to construct structured materials at the nanoscale. The compact organization of the molecules achieved in bent-core mesophases was transferred or induced to self-assemblies in the presence of water - even in the case of non-mesogenic dendrimers.

The subtle balance of molecule-molecule and molecule-solvent interactions regulated the morphology of the aggregates and these ranged from rods, non-twisted or twisted fibers, helical ribbons and nanotubes. The structure obtained was dependent on the choice of dendritic nucleus, the bent-core structure (from 5 to 3 aromatic ring systems), the lateral moiety and the length of the outer flexible tails. The rigid nature of the chemical structure controlled the type of molecular packing in the layers and allowed molecular conformational chirality to be transmitted to the supramolecular aggregates, despite achiral molecules were used. The short/long terminal chain lengths determined the type of curvature of the ribbons. Some of these self-assemblies are proposed as alternatives to the attractive and widely applied helical nanofilaments formed by bent-core mesogens, but, thanks to their amphiphilic nature, the novel compounds can be processed from solution. Moreover, the self-assembly process was studied in an effort to elucidate the aggregation mechanism of these bent-shaped amphiphiles and to define experimental protocols to provide high quality and homogeneous aggregates.

\section{Introduction}

Control of the self-assembly of small molecules to generate architectures with diverse shapes and dimensions by the supramolecular chemistry approach remains a topical, challenging and dynamic research field. Both the size and the morphology of the self-assembled structures influence the physical properties and potential applications in areas ranging from nanotechnology to biotechnology. ${ }^{[1-4]}$

Among the rich variety of morphologies, the formation of fibrous aggregates and their evolution from fibers and twisted fibrils to helical ribbons or tubes has recently attracted a great deal of attention due to its applicability in bottom-up nanoengineering. ${ }^{[5-7]}$ However, the prediction of the supramolecular structural features from combinations of noncovalent interactions is still in its infancy. A number of biological molecules ${ }^{[8-10]}$ have been proven to assemble to form fibers in variety of supramolecular systems. In these cases, the morphologies depend on the conformational characteristics at the monomeric level as well as the dynamics of the assembly process itself. Alternatively, other compounds that differ significantly from biological derivatives have also recently shown the ability to assemble into fibrillar and tubular systems, thus highlighting the molecular requirements for this type of

\footnotetext{
- Instituto de Ciencia de Materiales de Aragón, Departamento de Química Orgánica, Facultad de Ciencias, Universidad de Zaragoza-CSIC, Campus San Francisco, 50009-Zaragoza (Spain).

b. Address here.

c. Address here.

Electronic Supplementary Information (ESI) available: [details of any supplementary information available should be included here]. See DOI: 10.1039/x0xx00000x
}

behavior, ${ }^{[5,11-20]}$ where novel bent-core based molecules warrant particular attention.

From the supramolecular point of view, bent-shaped molecules have provided the newest class of thermotropic mesogens, which are known as 'bent-core liquid crystals' (BCLCs). The bent geometry of the molecules forces them to adopt a compact packing, thus restricting their rotational freedom and leading to a new family of polar mesophases with very attractive properties. ${ }^{[21-30]} A$ distinctive feature of this class of mesogen is the possibility of achieving not only polar organization but also spontaneous macroscopic chirality from achiral molecules, ${ }^{[31]}$ e.g., the SmCP, the B7 mesophase or the very attractive B4-like phases, which are also named HNF-type (HNFs, H $\mu F s$ and HLNCS) phases due to the formation of helical nanofilaments, ${ }^{[31-40]}$ chiral mesophases with great promise and strong optical activity. ${ }^{[41-45]}$ The mechanisms for the formation of all of these phases have not been fully explained, although different proposals have been published. [23,24,32,33,37,41-43,46-49] Furthermore, potential applications have been claimed for diverse functional purposes and this has provided inspiration for nanoengineers working on chiral separation, nonlinear optics and photovoltaics. ${ }^{[41-45,47,50,51]}$ Thus, BCLCs and their selfassembly have led to high expectations in supramolecular chemistry, materials science and nanotechnology.

Moreover, the ability of bent-shaped mesogens to promote supramolecular aggregates, including chiral objects, by selfassembly in a solvent has also been reported. Encouraged by the first publications by $\mathrm{Ho}$ and $\mathrm{Hsu}$ et al.[52,53] and also by reports of physical gel formation by bent-shaped molecules, ${ }^{\text {,54- }}$ 57] the possibility of creating bent-core assemblies that form supramolecular structures in solution is now a hot target to explore. However, despite the previously listed examples, this 
field is still taking its first steps and comprehensive studies to understand globally both the self-assembly in bulk and in solution are yet required. In order to underline this necessity, we recently published a review dedicated to mesogens that are also able to self-assemble in solvents. ${ }^{58}$

In the search for suitable new bent-core molecular designs capable to organising in solvents, dendritic liquid crystals may be appealing allies in sight of their well-known ability to give rise to functional materials. ${ }^{[59-65]}$ Side-chain liquid-crystalline dendrimers consisting of a dendritic core and different peripheral promesogenic substituents are able to self-assemble in bulk to generate micro- or nanosegregated domains ${ }^{[60,64,66-68]}$ through the segregation of polar and non-polar regions, with this phenomenon also providing the driving force for nanostructure formation in solution. ${ }^{[69-71]}$ In our previous work, ${ }^{[72]}$ ionic bent-core/poly(propyleneimine) PPI-based molecules were selected as new amphiphiles according to the generally proposed structural elements required for stable selfassembly to favor segregation effects in a solvent ${ }^{[5]}$ (Scheme 1 ). Interestingly, these complexes were able to self-assemble in water to yield the currently pursued supramolecular architectures, i.e., rods, spheres, non-twisted or twisted fibers, helical ribbons and tubules, depending on the molecular structure. This seeding finding confirmed that the compact packing that characterizes bent-core structures and induces the attractive bent-core mesophases could also be achieved in the presence of water, thus providing systems ranging from specific single objects to supramolecular structures. Despite the complexity of the molecules, the reported self-assembled structures showed several similarities to the morphologies of other supramolecular aggregates based on chiral surfactants or peptides. This discovery suggests a common mechanism for the formation of twisted-fibers, helical ribbons or tubules by helical coiling of tape-like aggregates. ${ }^{[2,5,73-78]}$ Furthermore, the stacking of twisted layers to form strands observed for this sort of aggregate resembles models proposed for the formation of helical nanofilaments through curvature deformation, which renders the abovementioned helical nanofilament phases reported for bent-core molecules. In this manner, these tubes and fibrous supramolecular architectures, when processed in solution, can be considered as innovative alternatives to both the tubular functional self-assembled systems from amphiphilic molecules ${ }^{[5]}$ and the attractive filaments of the BCLC-like phases and their potential functional applications.

Nevertheless, a subtle balance between molecule/solvent interactions seems to have a crucial influence in controlling the morphology of the aggregation and this can be tailored through appropriate molecular design. Thus, the marked influence of slight molecular variations on the nature of the resulting morphologies led us to carry out a systematic analysis of further design features and evaluate their effect on molecular aggregation with this sort of amphiphile, with particular emphasis on the formation of twisted and tubular structures. The aim was to obtain new insights into the parameters that govern the molecular nanostructured self-organizations, especially the induction of supramolecular chirality from this kind of achiral molecule.

In our previous study, ${ }^{[72]}$ the self-assembly of first generation PPI dendrimers with different flexible chain lengths were studied. Despite the good abilities of these derivatives to aggregate in solution, only one compound gave rise to helical nanostructures. In order to unravel the subtle balance of supramolecular interactions that govern the formation of helical ribbons and nanotubes, in this new work the molecular structure/self-assembly relationship potential was extended to structural aspects regarding both the bent-core structure itself (hydrophobic structure) and the dendritic moiety (hydrophilic head group) (Schemes 2-4). We report here the synthesis and self-assembly of novel PPI dendrimers that are capable of selfassemble in chiral nanostructures, including a new type of twisted fibers whose morphology reminds of the HNFs of the B4-type mesophase. Moreover, attention was also focussed on the influence and control of methodological procedures in terms of aggregate formation in water and to study further the self-assembly mechanism.

\section{Self-assembly processes}

\section{Molecular factors that affect the self-assembly of ionic bent- core amphiphiles}

A systematic structure/properties study was carried out with the aim of better understanding the potential of bent-core amphiphilic dendrimers to influence and control the morphology of the selfassembled objects. In the field of liquid crystals, these kinds of classical studies were essential to understand the parameters that govern liquid crystalline organizations, which in turn facilitated the development of mesomorphic materials. This approach is also expected to be helpful when applied to solvent-mediated selfassembling systems. Moreover, the systematic study of these materials can be helpful to establish similarities between the mechanisms of self-organisation both in bulk and in solvents.

With the above goal in mind, a series of ionic poly(propyleneimine)based dendrimers was studied. Most of the materials are reported here for the first time and they were synthesized for comparison with some previously described compounds. Structural modifications were applied to both the hydrophilic head (dendritic nucleus) and the hydrophobic part (bent-core moiety).

The previously reported terminology for this kind of ionic dendrimer was again used here to describe the new compounds and these are denoted by the dendritic core type [PPIg] (g: generation) followed by the nomenclature of the carboxylate-terminated bent-shaped moiety $[X-m-n]$, with the letters referring to the rigid nucleus $(X)$ and the inner $(\mathrm{m})$ and outer $(\mathrm{n})$ alkyl tails, respectively.

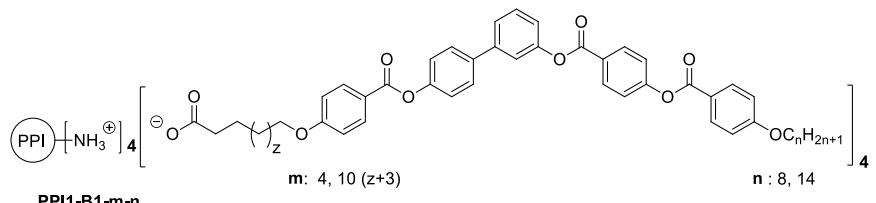

Scheme 1. Chemical structure and nomenclature of the four ionic bent-core/first generation PPI-based [PPI = poly(propyleneimine] dendrimers reported previously. ${ }^{[66,71]}$

In our previous research, ${ }^{72]}$ only first generation poly(propyleneimine) dendrimers (PPI1) bearing four bent-core units per molecule were used to obtain aggregates. The abilities of these dendrimers to self-assemble in water appeared to have the same origin as that of the liquid crystalline phases in bulk. Based on this finding, along with the previous results on the mesomorphism of higher generation dendrimers of this kind, ${ }^{[67]}$ the water-mediated self-assembly of more congested dendrimers was evaluated. Two different approaches were followed to increase the number of bentcore units per molecule up to eight while preserving the same bent- 
core structure (B1): on the one hand, the second generation of PPI was employed as the dendritic nucleus (compounds PPI2-B1-4-8 and PPI2-B1-10-14, both previously reported ${ }^{[67]}$ ) and, on the other hand, new dendritic systems (D1) that combined 2,2bis(hydroxymethyl)propionic acid [bis-MPA]-bent core-based dendrons with the first generation of PPI (PPI1-D1B1-4-8 and PPI1D1B1-10-14) (Scheme 2) were studied. In this investigation both short (m: 4, n: 8) and long ( $m: 10, n: 14)$ flexible tails were also considered, taking into account the dramatic influence of the interfacial region that links the hydrophilic head and the hydrophobic block $(\mathrm{m})$ or flexible outer chain lengths $(\mathrm{n})$ on the morphology of the aggregate, as revealed by previous results. ${ }^{[72]}$

Additionally, inspired by previous experience on bent-core liquid crystal self-assembly ${ }^{[36]}$ and in an effort to confirm and transfer learning to the less mature field of solvent-driven aggregation processes for these bent-shaped amphiphiles, the influence of further structural changes concerning the bent-core unit were addressed while retaining a first-generation PPI unit as the dendritic nucleus:

a) The effect of the incorporation of odd or even terminal chain lengths (from n: 7 to 10 carbon atoms) (Scheme 3).

b) The effect of modifications in the rigid bent-core structure (Scheme 4):

- Two isomers, namely phenylene (PPI1-Ph1-4-8) and benzoate (PPI1-Bz1-4-8) derivatives - both with five aromatic rings, as PPI1-B1-4-8 - are proposed to study the influence of the orientation of the ester linking groups. Moreover, the effect of reducing the number of aromatic rings on the supramolecular self-assembly of bent-core based dendrimers was also assessed in shorter analogues, PPI1-Bz4-8 and the very simple 1,3-phenylene derivative PPI1-Ph-48 , with four and just three aromatic rings, respectively.

- On the basis of very appealing and consistent recent results that demonstrate the strong ability of biphenyl-based bentcores to induce HNF-type mesophases, as described by Walba and Hegmann, $[31,34,39,40]$ compounds PPI1-Bi-4-8 and PPI1-Bi-4-10 with five aromatic rings and a biphenyl-ester lateral moiety were also included in our study.

All of the new PPI-based dendrimers were prepared according to the synthetic pathways shown in Schemes S1-S7. FT-IR and NMR data are consistent with the formation of the new dendrimers (see SI).

Liquid crystal self-assembly of new ionic dendrimers. The liquid crystalline organization of the new dendrimers was investigated by polarized optical microscopy (POM), differential scanning calorimetry (DSC), thermogravimetric analysis (TGA) and $\mathrm{X}$-ray diffraction (XRD). The properties are gathered in Table 1. In most cases, the bent-shaped carboxylic acids used to prepare ionic dendrimers did not form liquid crystalline phases or they showed a very narrow range of mesophase on cooling that difficulted their characterization (see SI). In contrast, upon incorporation of the bent compounds into an ionic dendrimer, most of the macromolecules formed mesophases, thus confirming the importance of the dendritic component to promote the supramolecular organization leading to liquid crystalline behavior. DSC thermograms of the ionic dendrimers on successive heating and cooling cycles presented reproducible melting transitions when the compounds were heated up to $10{ }^{\circ} \mathrm{C}$ below the clearing point. However, all compounds changed chemically when heated into the isotropic liquid, as reported for numerous other ionic dendrimers. ${ }^{[67,79]}$ In some cases, the pristine dendrimers exhibited a rich polycrystallinity that could not be recovered after melting. Single transitions from crystal to mesophase were observed from the second heating and these occasionally differed from the melting point determined in the first heating cycle and the transitions were significantly less energetic. Consequently, in the cases of the compounds that showed liquid crystalline behavior, the comparative discussion is based on melting and clearing temperatures from the second heating run of samples cooled from the mesophase. For those ionic dendrimers that did not show a mesophase, the clearing temperature corresponds to the first heating run.

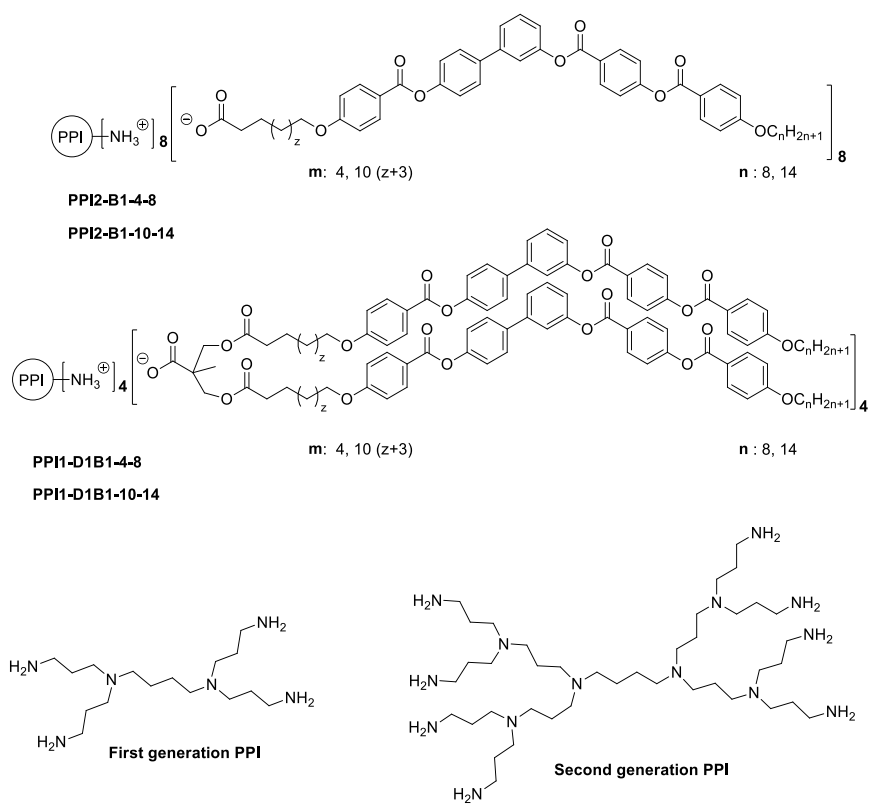

Scheme 2. Chemical structure and nomenclature of the new ionic bent-core/PPI-based dendrimers selected to analyze the influence of the dendritic moiety (hydrophilic head).

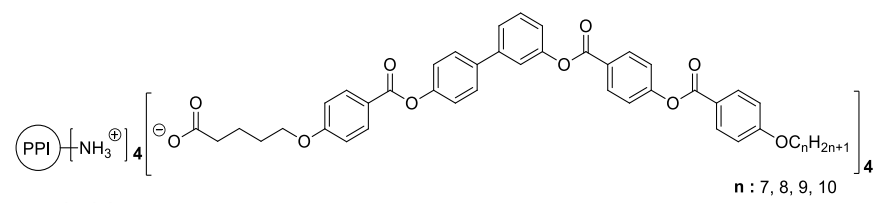

PPI1-B1-4-n

Scheme 3. Chemical structure and nomenclature of ionic bentcore/PPI-based dendrimers selected to analyze the influence of the terminal chain odd-even effect.

As far as the dendritic systems that contain eight bent-core units are concerned, mesomorphic behaviour of the second-generation PPI dendrimers (PPI2-B1-4-8 and PPI2-B1-10-14) ${ }^{[67]}$ in comparison to their previously described counterparts derived from the first generation leads one to conclude that the type of mesophase depends only on the flexible tail lengths and not on the generation of dendrimer. The incorporation of long alkyl chains ( $n: 10, m: 14)$ led to segregation from the aromatic core to give lamellar structures, whereas shorter terminal chains ( $n: 4, m: 8)$ favored intercalation of the molecules and the formation of columnar mesophases - a finding in good agreement with previous reports and with similar molecular packing proposed. ${ }^{[36,80,81]}$ On the other hand, the introduction of a bis-MPA dendron (PPI1-D1B1-4-8 and PPI1-D1B1-10-14) induced the formation of wide range $\mathrm{Col}_{\mathrm{r}}$ mesophases regardless of the length of the alkyl chains $(a=44.2, b=56.9$ for PPI1-D1B1-4-8; $a=58.3, b=$ 62.0 for PPI1D1B1-10-14), pointing out that the introduction of a 
flexible bis-MPA linker between the PPI dendrimer and the bent-core moieties favors the formation of columnar arrangements.<smiles>CCCCCCOC(=O)CCCOc1ccc(OC(=O)c2ccc(OC(=O)c3cccc(Oc4ccc(OC(=O)c5ccc(OCC)cc5)cc4)c3)cc2)cc1</smiles><smiles>CCCCCCCCCOC(=O)CCCOc1ccc(C(=O)Oc2ccc(C(=O)Oc3cccc(C(=O)Oc4ccc(OC(=O)c5ccc(OCC)cc5)cc4)c3)cc2)cc1</smiles>
PP11-Bz1-4-8<smiles>CCOC(=O)CCCOc1ccc(C(=O)Oc2cccc(C(=O)Oc3ccc(OC(=O)c4ccc(OCC)cc4)cc3)c2)cc1</smiles>

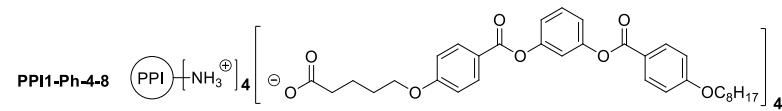

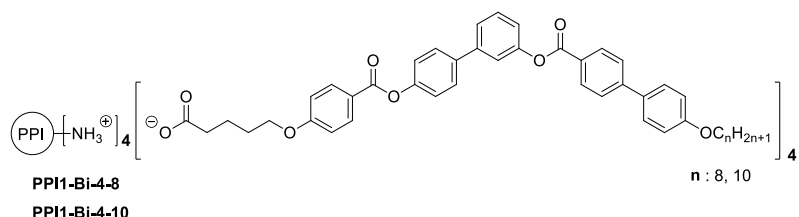

Scheme 4. Chemical structure and nomenclature of ionic bentcore/PPI-based dendrimers selected to analyze of the influence of the bent-core moiety.

Moving on to first-generation dendrimers, modification of the parity of carbon atoms in the terminal alkyl chain did not affect the type of mesomorphism of the ionic complexes. The four compounds, i.e. PPI1-B1-4-7, PPI1-B1-4-8, PPI1-B1-4-9 and PPI1-B1-4-10 formed Colr arrangements in the mesophase with similar structural parameters, as determined by XRD patterns (see SI). However, an odd/even effect was observed in the melting and clearing points. This is a typical feature that tunes most liquid crystal organizations but it is an aspect that is still under investigation. ${ }^{[82]}$ In the cases reported here, the odd-membered reduced both temperatures around $5^{\circ} \mathrm{C}$ in comparison to the even-membered counterparts.

With regard to the connectivity of the aromatic rings, the incorporation of an additional ester-linking group carried out modifications with respect to the PPI1-B1-4-8 structure. The influence of the orientation of the ester bond (isomers PPI1-Ph1-4-8 and PPI1-Bz1-4-8) was evaluated. The presence of a phenylene moiety (PPI1-Ph1-4-8) led to the formation of a Col ${ }_{r}$ phase $(a=63.0$ $\AA$ and $b=55.2 \AA$ ) over a wide temperature range and this was similar to the phase of PPI1-B1-4-8. In contrast, the benzoate derivative seemed to hinder mesomorphism as PPI1-Bz1-4-8 only showed a mesophase over a few degrees and this was difficult to identify. This behavior can be attributed to the unsymmetrical distribution of the dipoles arising from the ester linking group since symmetric dispositions favor core-core interactions, which stabilize the mesophase. ${ }^{[36]}$ Likewise, in spite of the high tendency for dendrimers to stabilize liquid crystal organizations, a reduction in the number of aromatic rings disfavors mesophase formation and the shortest analogs (PPI1-Ph-4-8 and PPI1-Bz-4-8) melt directly from the crystal below $120^{\circ} \mathrm{C}$ to the isotropic liquid. Remarkably, the crystal-isotropic liquid transition enthalpy for PPI1-Ph-4-8 has a very low energy, especially when compared with the rest of dendrimers that do not show a mesophase, and this indicates that three-ring bent-core structures give rise to low-order crystallinity of the solid due to poor stacking within the cores.

\begin{tabular}{|c|c|c|c|}
\hline Dendrimer & $\begin{array}{l}\text { Phase transition } \\
{\left[{ }^{\circ} \mathrm{C}\left(\Delta \mathrm{H}, \mathrm{kJ} \mathrm{mol}^{-1}\right)\right]^{\mathrm{alb})}}\end{array}$ & $\begin{array}{l}\text { Morphology } \\
\text { of aggregates }\end{array}$ & $\begin{array}{l}\text { Dimensions of } \\
\left.\text { aggregates }{ }^{j}\right)\end{array}$ \\
\hline PPI1-B1-4-8 C) & Cr 105 (13.6) Colr 144 (6.9) I & $\begin{array}{l}\text { Helical ribbons } \\
\text { and nanotubes } \\
\text { h) }\end{array}$ & $\begin{array}{c}L: \mu \mathrm{m}, \\
w \approx 300-450 \mathrm{~nm}, \\
\Phi \approx 400-600 \mathrm{~nm}, \\
d: 5.3 \mathrm{~nm}\end{array}$ \\
\hline PP12-B1-4-8 d) & Cr 76 (26.6) Colr 138 (30.6) I & Undefined ${ }^{i)}$ & - \\
\hline PPI1-D1B1-4-8 & Cr $93(50.6)$ Col $_{\text {r }} 158$ (102.6) I & Rods & $\begin{array}{c}\mathrm{L} \approx 100-150 \mathrm{~nm}, \\
\mathrm{w} \approx 30 \mathrm{~nm}\end{array}$ \\
\hline PP12-B1-10-14 d) & 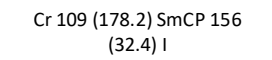 & Undefined") & - \\
\hline PPI1-D1B1-10-14 & Cr 106 (99.1) Colr 141 (10.6) I & Undefined ") & - \\
\hline PPI1-B1-4-7 & Cr99 (14.7) Colr 140 (24.8) I & Nanotubes & $\begin{array}{c}\text { L: } \mu \mathrm{m}, \\
\Phi \approx 500-750 \mathrm{~nm}\end{array}$ \\
\hline PP11-B1-4-9 & Cr 100 (9.1) Colr 141 (13.5) I & $\begin{array}{c}\text { Non-twisted } \\
\text { ribbons }\end{array}$ & $\begin{array}{c}\mathrm{L:}: \mu \mathrm{m}, \\
\mathrm{w} \approx \underset{\mathrm{nm}}{200 \mathrm{~nm}-600}\end{array}$ \\
\hline PPI1-B1-4-10 & Cr 105 (12.9) Colr 144 (16.4) I & $\begin{array}{c}\text { Non-twisted } \\
\text { ribbons }\end{array}$ & $\begin{array}{c}\text { L: } \mu \mathrm{m}, \\
w \approx 200-400 \mathrm{~nm}\end{array}$ \\
\hline PPI1-Ph1-4-8 & Cr $103(12.0)$ Colr $_{r} 150$ (17.7) I & $\begin{array}{l}\text { Helical ribbons } \\
\text { and nanotubes }\end{array}$ & $\begin{array}{c}\text { L: } \mu \mathrm{m}, \\
\mathrm{w} \approx 160-210 \mathrm{~nm}, \\
\Phi \approx 180-280 \mathrm{~nm}\end{array}$ \\
\hline PPI1-Bz1-4-8 & Cr 137 M $145(92.7)^{n)}$ & $\begin{array}{l}\text { Non-twisted } \\
\text { fibers } \\
\text { and ribbons }\end{array}$ & $\begin{array}{c}\text { L: } \mu \mathrm{m}, \\
w \approx 40-200 \mathrm{~nm}\end{array}$ \\
\hline PPI1-Bz-4-8 & $\left.\operatorname{Cr} 117(162.0)\right|^{e)}$ & $\begin{array}{l}\text { Non-twisted } \\
\text { ribbons }\end{array}$ & $\begin{array}{c}\mathrm{L:}: \mu \mathrm{m}, \\
\mathrm{w} \approx 300-500 \mathrm{~nm}\end{array}$ \\
\hline PPI1-Ph-4-8 & $\left.\operatorname{Cr} 112(15.7)\right|^{e)}$ & $\begin{array}{c}\text { Non-twisted } \\
\text { fibers }\end{array}$ & $\begin{array}{c}\mathrm{L}: \mu \mathrm{m}, \\
w \approx 20-30 \mathrm{~nm}\end{array}$ \\
\hline PPI1-Bi-4-8 & Cr 122 (39.1) HNF 165 8) I & Twisted fibers & $\begin{array}{c}\text { L: } \mu \mathrm{m}, \\
\mathrm{w} \approx 20 \mathrm{~nm}, \\
\mathrm{~h}: 60-70 \mathrm{~nm}, \\
d: 5-6 \mathrm{~nm}\end{array}$ \\
\hline PPI1-Bi-4-10 & Cr $112(12.2)$ HNF 168 (48.8) I & Twisted fibers & $\begin{array}{c}\mathrm{L}: \mu \mathrm{m}, \\
\mathrm{w} \approx 25 \mathrm{~nm}, \\
\mathrm{~h}: 70-80 \mathrm{~nm}\end{array}$ \\
\hline
\end{tabular}

Table 1. Transition temperatures for the ionic bent-core based dendrimers and morphologies of the aggregates formed in the presence of water. a) Data from DSC second heating scan at a scanning rate of $10{ }^{\circ} \mathrm{C} / \mathrm{min}$, of cooled samples from mesophase. Temperature data at the maximum of the peaks. ${ }^{\text {b) }} \mathrm{Cr}$ : Crystal; SmCP: polar smectic $\mathrm{C}$ mesophase; Colr: rectangular columnar mesophase; M: unidentified mesophase, HNF: helical nanofilament-type mesophase, I: isotropic liquid. c) Liquid crystalline properties reported previously in reference 72 . d) Liquid crystalline properties reported previously in reference 67 . e) Data from DSC first heating scan, at a scanning rate of $10^{\circ} \mathrm{C} / \mathrm{min}$. T data at the maximum of the peaks. f) Combined enthalpies. g) Transition determined by POM. h) Aggregates in water previously reported in reference 72 . i) The aggregate precipitates and showed an undefined morphology by TEM. j) L: length, $\Phi$ : diameter of nanotubes, $w$ : width of fibers or ribbons, h: half pitch, $d$ : layer spacing estimated from TEM. 
Finally, the introduction of biphenyl-ester moieties as lateral structures as for PPI1-Bi-4-8 and PPI1-Bi-4-10, interestingly led to the formation of an alternative mesophase to the $\mathrm{Col}_{\mathrm{r}}$ and SmCP. The Xray diffraction pattern of the liquid crystal phase showed multiple periodic reflections in the low angle region, that are attributed to a lamellar order with a layer spacing of $80.0 \AA$ for PPI1-Bi-4-8 and 83.0 $\AA$ A for PPI1-Bi-4-10, and several diffuse peaks in the wide-angle region. The data are consistent with a hexagonal in-plane order within the lamellae ${ }^{[33]}$ and this is coherent with the formation of a helical nanofilament phase (or B4 phase) that vitrifies at room temperature. Unfortunately, chiral domains could not be clearly observed by POM. Interestingly, these new compounds join the previous but scarce examples that show desirable HNF-like organizations and this highlights the ability of biphenyl lateral moieties to promote these promising phases. ${ }^{[34,38-40]}$

Self-assembled aggregates of ionic dendrimers in water. The solvent-mediated aggregation (THF and water) of the ionic dendrimers was carried out using a standard procedure ${ }^{[70,72]}$ as follows: the ionic dendrimer was dissolved in THF at $0.5 \mathrm{wt} \%$, with the exception of PPI1-Bi-4-8, which was prepared at $0.2 \mathrm{wt} \%$ due to the low solubility of the dendrimer. The aggregation process was determined by turbidity measurements. Milli- $Q$ water was slowly added to the THF solution (5-10 mL of water per minute to $1.6 \mathrm{~mL}$ of dendrimer solution) with gentle shaking. The optical density (turbidity) of the solution was measured after every addition of water at a wavelength of $650 \mathrm{~nm}$ using a quartz cell (path length $=1$ $\mathrm{cm}$ ) with a Unicam UV/vis spectrophotometer, with the solution left to equilibrate until the turbidity value remained constant. The cycle of water addition, equilibration and turbidity measurement was continued until the critical aggregation concentration was exceeded, which was detected by a sudden change in the slope of the optical density function in relation to the volume of water added and the increase in turbidity upon further addition of water was very small and a plateau was reached. The solution was then dialyzed against water to remove THF using a Spectra/Por regenerated cellulose membrane with a molecular weight cut-off of $1000 \mathrm{kDa}$. When the addition of water caused the precipitation of a proportion of the material, the solid was removed and the study was continued with the stable solution. Samples were studied by transmission electron microscopy (TEM) (Figure 1) and this provided convincing evidence for the formation of different aggregates depending on the chemical structure of the ionic molecule (Table 1).

Remarkably, most of the ionic dendrimers are suitable molecules to self-organize as nanostructures in solution upon the addition of water (a poor solvent). Exceptions are most of the more congested dendrimers (PPI2-B1-4-8, PPI2-B1-10-14 and PPI1-D1B1-10-14), which contain eight bent-core units, whose aggregates promptly precipitated and showed an ill-defined morphology by TEM. Only PPI1-D1B1-4-8 led to the formation of well-defined small rods (100$150 \mathrm{~nm}$ in length and $30 \mathrm{~nm}$ in width), as shown in Figure 1a. These results indicate that, in spite of the tendency for these molecules to form mesophase assemblies in bulk, more congested dendritic structures - such as those based on second generation PPI or bisMPA - do not favor the formation of defined aggregates in solution, suggesting different molecular conformations (extended vs folded) of the ionic dendrimers in bulk or in solution. Furthermore, these results could also support the on/off switching role proposed for the head group of amphiphiles to promote the formation of helix-based tubes $^{[5]}$ versus the tuning role of the hydrophobic part. The shape of the hydrophilic group and its orientation relative to the hydrophobic structures seems to be essential for the assembly of these congested molecules to build either twisted fibers or helix-based nanotubes, thus limiting the obtaining of these nanostructures to first generation PPI dendrimers directly grafted to four bent-core structures.

If we now focus on the modifications of the hydrophobic structures of the bent-shaped amphiphiles, the influence of flexible terminal tails will be discussed first. It can be seen from the results in Table 1 that all the dendrimers with four bent-core units allowed the preparation of defined nanostructures.

Interestingly, unlike in the case of liquid crystal behavior, the modification of the length of the aliphatic chains provoked marked differences in the morphology of the aggregates of PPI1-B1-4-7, PPI1B1-4-8, PPI1-B1-4-9 and PPI1-B1-4-10 (Figures 1b-e). Seven- and eight-membered terminal chains favored the formation of helical ribbons and nanotubes (500-750 nm in width for PPI1-B1-4-7 and 300-450 nm in width for PPI1-B1-4-8), whereas non-twisted ribbons were obtained when nine- and ten-carbon chains were employed (200-600 nm in width for PPI1-B1-4-9 and 200-400 nm in width for PPI1-B1-4-10). These results reveal a striking contribution of the flexible tails, as tubular architectures were only obtained for the derivatives with shorter terminal alkyl chains, thus reinforcing the strong influence of the chain length on the morphology of the aggregates and highlighting the existence of a subtle balance of supramolecular forces that enable the formation of the helical nanostructures in solution. This equilibrium seems to be much more fragile than in the case of liquid crystalline organisations.

On considering the role of the most unique fragment of the bentcore hydrophobic structures, i.e., the rigid bent-core, TEM images of aggregates of PPI1-Bz1-4-8, PPI1-Bz-4-8 and PPI1-Ph-4-8 (Figures 1fh) showed the formation of systems ranging from long fibrillar objects that are several micrometres in length and with widths ranging from $20-30 \mathrm{~nm}$ in the case of PPI1-Ph-4-8 (3 aromatic rings) to wider fibers of hundreds of $\mathrm{nm}$ in width for the benzoate-based dendrimers PPI1-Bz-4-8 (4 aromatic rings) and PPI1-Bz1-4-8 (5 aromatic rings). Interestingly, these amphiphilic dendrimers, including those with small rigid cores, all are suitable for the formation of fibrillar structures from water, although these compounds failed to promote liquid crystalline organizations in bulk. More interestingly, aggregates formed by PPI1-Ph1-4-8 (5 aromatic rings) again showed tubular morphologies and these nanotubes resembled the ones already obtained with PPI1-B1-4-7 and PPI1-B14-8. However, they had a remarkably smaller diameter (150-300 $\mathrm{nm})$. This finding demonstrated the possibility of tuning the dimensions of the nanostructures through molecular design modifications. Nevertheless, although tubular morphologies were obtained for three of the ionic dendrimers, these attractive structures with high potential remain quite elusive and exclusive due to the delicate balance of supramolecular forces that affect their formation.

Intriguingly, the comparison of the aggregation abilities of PPI1-B14-8, PPI1-Ph1-4-8 and PPI1-Bz1-4-8 (all with 5 aromatic rings) indicates that structural aspects that affect mesophase assembly also seem to play a crucial role in water-assisted aggregation. Unlike PPI1B1-4-8 and PPI1-Ph1-4-8, that self-assembled in nanotubes, PPI1Bz1-4-8, in which the ester bond is inverted and provided a very narrow range of mesophase in bulk, again did not favor higher levels of self-aggregation in solution and only non-twisted ribbons were stabilized. 

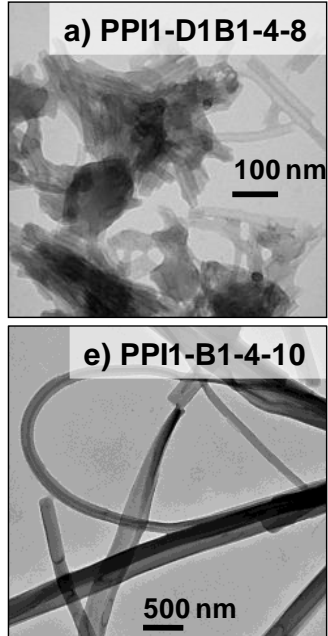

$500 \mathrm{~nm}$

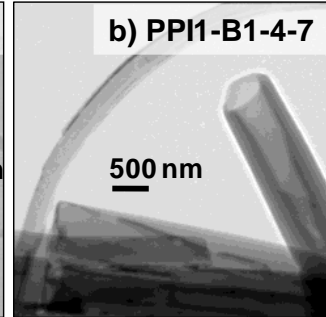

f) PPI1-Bz1-4-8

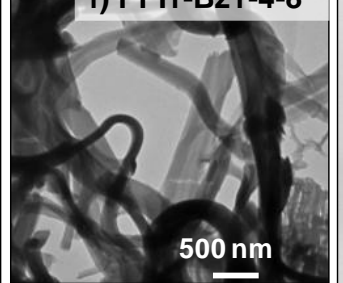

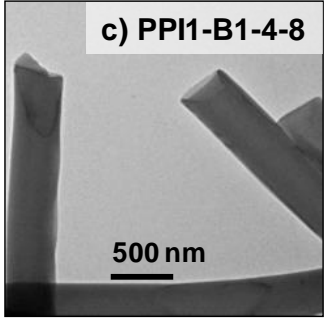

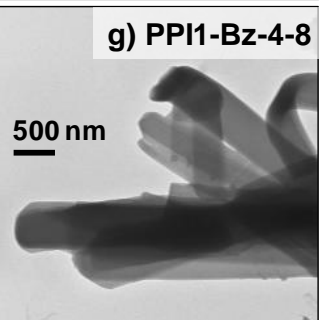

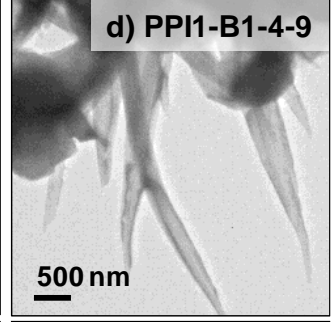

h) PPI1-Ph-4-8

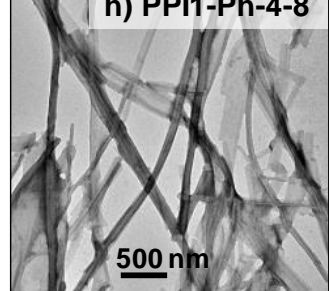

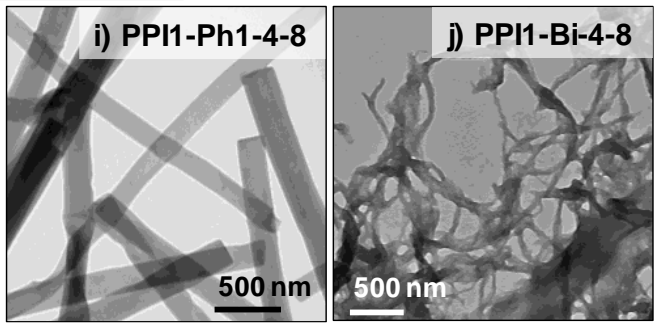

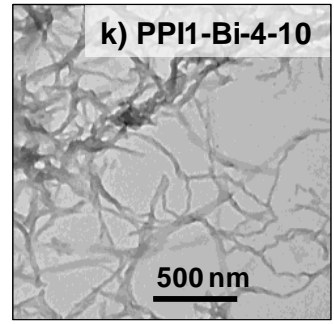

Figure 1. Transmission electron microscopy (TEM) images of aggregates of the ionic dendrimers in water: rods (a), nanotubes (b, c and i), non-twisted fibers and ribbons ( $d-h)$, and twisted fibers ( $\mathrm{j}$ and $\mathrm{k}$ ).

Finally, different attractive chiral fibrillar arrangements were achieved from dendrimers PPI1-Bi-4-8 and PPI1-Bi-4-10. In a similar way to the liquid crystalline behavior, the introduction of a lateral biphenyl unit on the dendrimers promoted novel types of supramolecular organization in solution; twisted fibers (several micrometers in length and around $20-25 \mathrm{~nm}$ in width) with a pitch in the range of 60-80 $\mathrm{nm}$ were generated by self-assembly, as observed in the TEM images depicted in Figures $1 \mathrm{j}$ and $1 \mathrm{k}$. Unlike flat tapes formed by other ionic dendrimers, the fibers of PPI1-Bi-4-8 and PPI1$\mathrm{Bi}-4-10$ seemed to be thicker and had a morphology similar to that observed in FFTEM images of HNF phases in bulk. Typically, HNFs reported are around $25-30 \mathrm{~nm}$ in width and have a helical pitch of $100 \mathrm{~nm} \cdot{ }^{[31,33]}$ These values are in good agreement with those observed for the twisted fibers of PPI1-Bi-4-8 and PPI1-Bi-4-10. All of these findings, together with the fact that that these materials showed the HNFs mesophase in bulk, led us to believe that PPI1-Bi4-8 and PPI1-Bi-4-10 aggregates may be considered as a sort of helical nanofilament in solution.

\section{Controlling the formation of chiral nanofibers and nanotubes in solution}

To understand the step-formation of complex supramolecular selfassemblies and to achieve sufficient control to obtain high-quality specimens are both hot topics in solvent-mediated nanostructured aggregates as this provides a way to speed up their functional applications. For this reason, in addition to the control of the morphologies by molecular design, further targets were pursued in our work concerning the preparation of these nanoobjects. With a particular focus on the appealing nanotubes, several variations in the standard protocol for supramolecular synthesis detailed above were also considered in our research. These studies were focused initially on PPI1-B1-4-8 as a model.

Firstly, the influence that the amount of water had on the aggregation process was assessed. For this purpose, samples were analyzed before and after the appearance of turbidity upon addition of water. As can be seen from the TEM experiments, at lower water proportions PPI1-B1-4-8 is already able to self-assemble to form twisted fibrillar aggregates (Figure 2a) and fibers can evolve to helical nanoribbons and nanotubes after successive additions of water (Figures $2 \mathrm{~d}$-e). This interesting behavior led us to study the evolution of the twisted fibers with time. Solutions at low water proportions were studied by TEM for different time periods, i.e., once the equilibration of the solution had occurred ( $\mathrm{t}$ : 0 ), after 1 day and after 19 days (Figures $2 a-c$ ). Once again, the initial twisted fibers assembled further to form long helical ribbons up to $450 \mathrm{~nm}$, which collapsed to give empty nanotubes after 19 days of incubation.

Additionally, the effect of complete removal of the organic solvent through dialysis was also checked. Dialysis provided cleaner, more homogenous and highly populated samples of nanotubes, although it also led to partial precipitation of the aggregates (Figures $2 \mathrm{~d}$ and 2e). Moreover, when samples were sonicated for a few minutes after dialysis, the amount and quality of homogeneous nanotubes increased (Figure 2f). Thus, these experiments highlighted the importance of the control of parameters such as time evolution, dialysis and sonication on the supramolecular synthesis by selfassembly and helped to define an optimum procedure in which both stabilization time and dialysis were considered essential.

The results discussed above were replicated with samples of PPI1Ph1-4-8 both before and after dialysis, as shown by TEM. It can be seen from Figure 3 that before dialysis PPI1-Ph1-4-8 had formed helical nanoribbons of several microns in length and around 160-210 
$\mathrm{nm}$ in width, with both left- and right-handed helices, whereas after dialysis the nanoribbons closed on themselves to form empty tubules of 180-280 nm in diameter, that is, analogous to - but thinner than - those observed previously for PPI1-B1-4-8. The diameter of the tubular structures is directly related to the width of the helical ribbons. In this manner, bent-core dendrimers that are able to assemble in more extensive layers give rise to wider helical tapes and therefore bigger nanotubes.
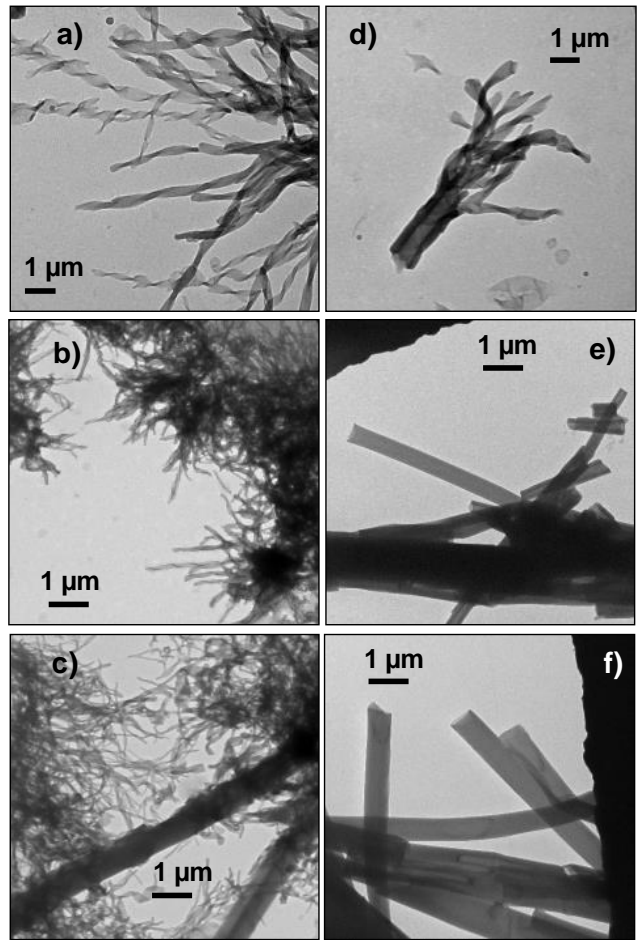

Figure 2. TEM images of PPI1-B1-4-8 at different conditions to evaluate the effect of procedure parameters in the morphology of the aggregates. Left: From non-dialyzed solutions before the appearance of turbidity, at a) t: 0, b) t: 1 day, and c) t: 19 days. Right: From solutions after the sudden increase of turbidity: d) without dialysis and sonication processes, e) after dialysis, and f) after dialysis and sonication.

Based on these observations we propose packing models similar to those previously reported by us for the formation of tubes from PPI1B1-4-8 (Figure 3). ${ }^{[72]}$ The folded dendrimers arrange in layers with the aromatic cores compacted within the structure and the hydrophilic parts facing outwards towards the water, thus leading to layered fibers. The progressive addition of water (or the equilibration over time) favors lateral growth steps and the widening of the tapes causes the transition from twisted fibers with Gaussian curvature to helical ribbons with mean curvature, which eventually close on themselves to form nanotubules. ${ }^{[5,75]}$ This process resembles the mechanisms reported previously for simpler systems such as amino acid-based amphiphiles. ${ }^{[83-85]}$

Similar preparation methodologies and studies were carried out for PPI1-Bi-4-8. TEM images of aggregates before dialysis showed twisted fibers consisting of regular strands that were about $5 \mathrm{~nm}$ wide (Figure 4). This layer spacing is similar to those observed in FFTEM images of HNF phases in bulk for common bent-core molecules, ${ }^{[31,33]}$ once again supporting our assumption that ionic dendrimers self-assemble in solution in a similar way to the lamellar bent-core mesophases. Since the layer spacing is according to half the estimated length of the extended molecule (PPI1-Bi-4-8, around $100 \AA$, estimated with ChemSketch), we propose that the ionic dendrimer folds to expose the hydrophilic PPI moiety to the water when forming layers. The fibers seem to be formed by around 5-6 layers of the folded dendrimer, which regularly twists with a pitch of 60-80 $\mathrm{nm}$. This supramolecular arrangement is very similar to that of the helical nanofilaments reported for a variety of B4-type mesophases, including the HNF phase, also identified for PPI1-Bi-4-8 and PPI1-Bi-4-10 in the mesophase in bulk, albeit with a larger layer spacing consistent with an extended molecular disposition.

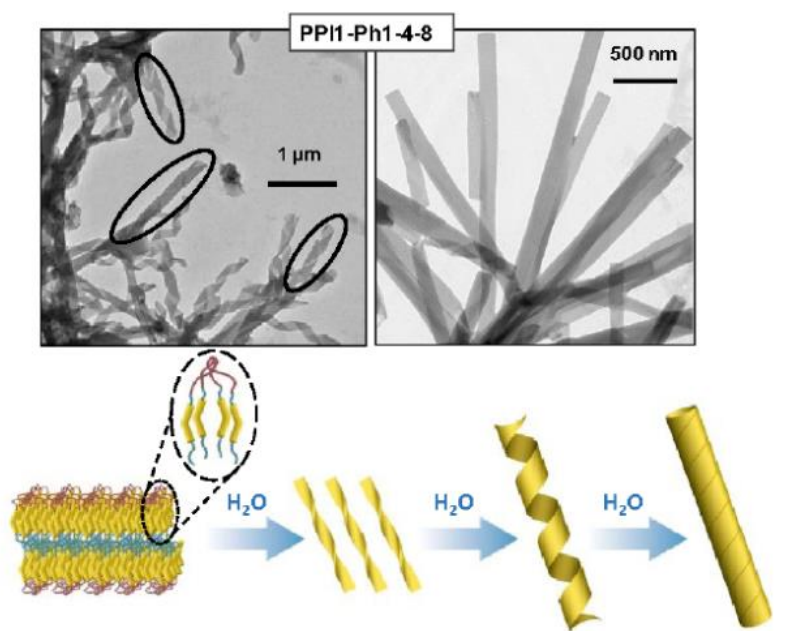

Figure 3. Proposed self-assembly mechanism for PPI1-Ph1-4-8. Top: TEM images of the nanostructures before dialysis (left) showing both left- and right-handed helices, which collapse to form nanotubes very homogenous after dialysis (right). Down: proposed mechanism for the formation of nanotubes upon water addition.

In this respect, it is planned to carry out further studies to compare the molecular self-assembly of these molecules both in the mesophase and the fibrillar aggregates. Nevertheless, based on the current results, we propose a similar origin and mechanism to render the twist for both types of self-assembly. In a comparable manner to the BCLCs, the rigid bent structure controls the molecular packing and causes adjacent ester groups to rotate at different angles relative to each other, thereby resulting in the formation of the optimum molecular geometry with a molecular twist and spontaneous chiral symmetry breaking. Due to this tilt, the lamellae suffer compression tension that forces them to twist. This twisting results in the formation of either the helical nanofilaments that give the name to the mesophase in bulk or the fibrillar aggregates in the presence of a solvent (Figure 4).

The molecular conformational chirality of these particular bentshaped amphiphiles can therefore be successfully transferred from the molecular level to supramolecular aggregates through different self-assembly systems. However, the reason why some twisted fibers either evolve or don't to form wider ribbons, and have a saddle-like shape whereas ribbons have a cylindrical curvature leading to tubes, is still a matter of debate that continues to motivate both experimental and theoretical studies. ${ }^{[5,73-75,77,83-87]}$ Interestingly, it has been proposed that the formation of twisted fibers versus helical ribbons is related to the lateral assembly width of the tapes, which is typically (but not always) influenced by the length of the alkyl tails, that form the bilayer assembly and control the fluid or crystalline organizations. ${ }^{[5,72,74,86]}$ This proposal could so support some of our results to a certain extent. Nevertheless, for these novel bent-shaped 
amphiphiles the overall molecular structure also seems to play a key role in directing the resultant shape of the self-assembled morphology.

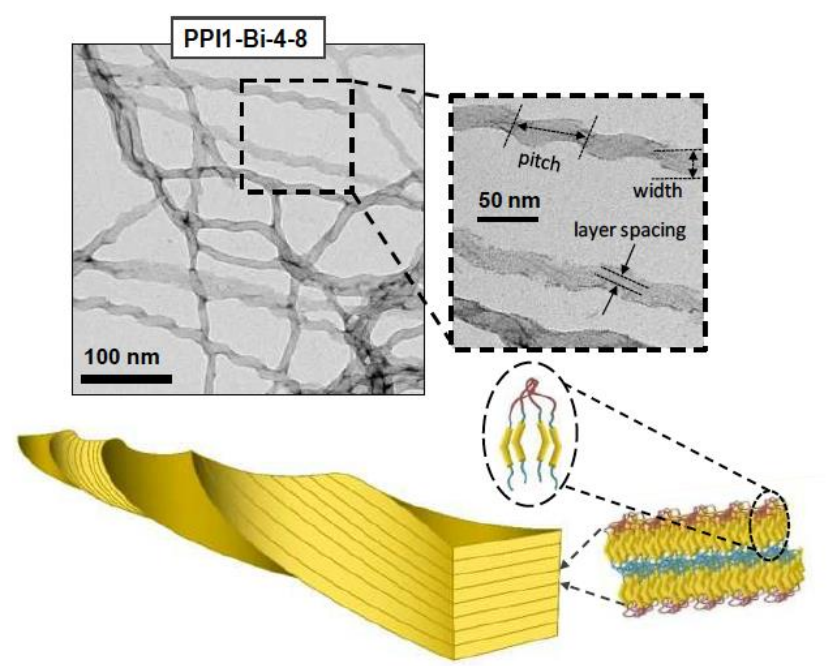

Figure 4. Proposed self-assembly mechanism for PPI1-Bi-4-8. Top: TEM image of the nanostructures before dialysis. Down: proposed mechanism for the formation of twisted-fibers similar to helical nanofilaments reported to form HNF mesophase.

\section{Conclusions}

The results obtained in this study demonstrate that ionic bent-core dendrimers, due to their amphiphilic nature, are suitable to allow the assembly of bent-shaped units not only in bulk but also in water. This possibility provides useful materials that are structured at the nanoscale. The compact packing that characterizes bent-core structures, as demonstrated in BCLCs, can be attractively achieved in the presence of water, even with non-mesogenic dendrimers. Nevertheless, a subtle balance between molecule/solvent interactions regulates the morphology of the aggregation, which ranges from rods, non-twisted or twisted fibers, helical ribbons and nanotubes depending on the chemical structure of the dendrimers. From the molecular design point of view, the appropriate selection of the dendritic nucleus as the hydrophilic head, the hydrophobic bent-core structure (with 5-3 aromatic rings systems) and the combination of inner and the outer flexible tails allows the morphology of the aggregates to be modulated, including the formation of chiral nanoscale objects formed by achiral molecules. The rigid bent-core structure controls the type of molecular packing and provides molecular conformational chirality that can be transferred to the supramolecular aggregates; the terminal chain lengths and the rigid lateral structures are mainly responsible for the type of curvature of the fibrillar-layered organizations (saddle-like, cylindrical or none).

These new results consolidate the attractive potential of supramolecular self-assemblies for the design of new nanostructured materials in a bottom-up strategy. Such materials are envisaged to be of interest for nanotechnology and advanced materials applications. With this aim in mind, a standard experimental protocol including dialysis against water and sonication processes have been developed to provide high quality homogeneous aggregates. Furthermore, wide-ranging attempts to evaluate approaches to control the self-assembly of these new amphiphiles, in particular in the route to tubular objects, is now an open area for further research.

Besides, it would be possible to combine a variety of innovative functional motifs (photoresponsive, NLO-active, etc.) compatible with the assembly of bent-core structures as well as the properties reported for BCLCS in these new supramolecular self-assembled architectures. Furthermore, from our point of view, these selfassembled systems can be considered as innovative alternatives to the helical nanofilaments reported to form a variety of B4-type mesophases (HNFs, H $\mu$ Fs and HLNCs), but with these new materials processed from solution. In this way, very interesting hybrid systems recently reported to be suitable for HNF could be certainly evaluated for these new aggregates, thus potentially providing great potential in the route towards functional supramolecular nanostructured materials.

\section{Conflicts of interest}

There are no conflicts to declare.

\section{Acknowledgements}

The authors greatly appreciate financial support from the Spanish Government (MINECO-FEDER project MAT2015-66208C3-1-P, PGC2018-093761-B-C31) and the Government of Aragon (GA) (project E47_17R). We are also grateful to Juan de la Cierva-MICINN and JAEDOC-CSIC (N.G.), GA (M.C.) and BES2016-078753 MINECO-FEDER (M. C-V.) fellowship programs for support. Thanks are given to the nuclear magnetic resonance, mass spectrometry, and thermal analysis services of the ICMA (Uni. Zaragoza-CSIC) and the LMA from INA (Uni. Zaragoza) for TEM equipment. M. C-V. thanks Pablo Monti for his help with the $3 \mathrm{D}$ design of the assembling models.

\section{References}

[1] J. M. Lehn, Ed., Supramolecular Chemistry: Concepts and Perspectives, Wiley-VCH, Weinheim, 2006.

[2] A. Sorrenti, O. Illa, R. M. Ortuño, Chem. Soc. Rev. 2013, 42, 8200-8219.

[3] S. I. Stupp, L. C. Palmer, Chem. Mater. 2014, 26, 507-518.

[4] D. B. Amabilino, D. K. Smith, J. W. Steed, Chem. Soc. Rev. 2017, 46, 2404-2420.

[5] T. G. Barclay, K. Constantopoulos, J. Matisons, Chem. Rev. 2014, 114, 10217-10291.

[6] M. S. Ekiz, G. Cinar, M. A. Khalily, M. O. Guler, Nanotechnology 2016, 27, 402002.

[7] S. Ling, D. L. Kaplan, M. J. Buehler, Nat. Rev. 2018, 3, 1-15.

[8] A. N. Moore, J. D. Hartgerink, Acc. Chem. Res. 2017, 50, 714-722.

[9] L. Guyon, E. Lepeltier, C. Passirani, Nano Res. 2018, 11, 
$2315-2335$.

[10] F. Benn, N. E. C. Haley, A. E. Lucas, E. Silvester, S. Helmi, R. Schreiber, J. Bath, A. J. Turberfield, Angew. Chem. Int. Ed. 2018, 7687-7690.

[11] H.-L. Sun, Y. Chen, X. Han, Y. Liu, Angew. Chem. Int. Ed. 2017, 56, 7062-7065.

[12] E. Cohen, H. Weissman, I. Pinkas, E. Shimoni, P. Rehak, P. Král, B. Rybtchinski, ACS Nano 2018, 12, 317-326.

[13] M. Hifsudheen, R. K. Mishra, B. Vedhanarayanan, V. K. Praveen, A. Ajayaghosh, Angew. Chem. Int. Ed. 2017, 56, 12634-12638.

[14] H. Zhang, A. Mourran, Nano Lett. 2017, 17, 2010-2014.

[15] S. Wu, Y. Li, S. Xie, C. Ma, J. Lim, J. Zhao, D. S. Kim, M. Yang, D. K. Yoon, M. Lee, et al., Angew. Chem. Int. Ed. 2017, 56, 11511-11514.

[16] H. L. K. Fu, S. Y. L. Leung, V. W. W. Yam, Chem. Commun. 2017, 53, 11349-11352.

[17] Y. Wang, X. Li, F. Li, W. Y. Sun, C. Zhu, Y. Cheng, Chem Commun. 2017, 53, 7505-7508.

[18] T. Wolf, A. Niazov-Elkan, X. Sui, H. Weissman, I. Bronshtein M. Raphael, H. D. Wagner, B. Rybtchinski, J. Am. Chem. Soc. 2018, 140, 4761-4764.

[19] H. L. Au-Yeung, S. Y. L. Leung, V. W. W. Yam, Chem. Commun. 2018, 54, 4128-4131.

[20] X. Liu, H. Li, Y. Kim, M. Lee, Chem. Commun. 2018, 54, 3102-3105.

[21] G. Pelzl, S. Diele, W. Weissflog, Adv. Mater. 1999, 11, 707724.

[22] M. Hird, Liq. Cryst. Today 2005, 14, 9-21.

[23] H. Takezoe, Y. Takanishi, Jpn. J. Appl. Phys. 2006, 45, 597625.

[24] R. A. Reddy, C. Tschierske, J. Mater. Chem. 2006, 16, 907961.

[25] G. Pelzl, W. Weissflog, Thermotropic Liquid Crystals: Recent Advances, Springer, Berlin, 2007.

[26] A. Jakli, C. Bailey, J. Harden, Thermotropic Liquid Crystals: Recent Advances, Springer, Berlin, 2007.

[27] A. Eremin, A. Jákli, Soft Matter 2013, 9, 615-637.

[28] A. Jákli, Liq. Cryst. Rev. 2013, 1, 65-82.

[29] C. Tschierske, Angew. Chem. Int. Ed. 2013, 52, 8828-8878.

[30] J. W. Goodby, P. J. Collings, T. Kato, H. Gleeson, C. Tschierske, P. Raynes, Eds. , Handbook of Liquid Crystals Vol. 4, Wiley-VCH, Weinheim, 2014.
[31] K. V. Le, H. Takezoe, F. Araoka, Adv. Mater. 2017, 29, 1-21.

[32] D. R. Link, G. Natale, R. Shao, J. E. Maclennan, N. A. Clark, E. Körblova, D. M. Walba, Science 1997, 278, 1924-1927.

[33] L. E. Hough, H. T. Jung, D. Krüerke, M. S. Heberling, M. Nakata, C. D. Jones, D. Chen, D. R. Link, J. Zasadzinski, G. Heppke, et al., Science 2009, 325, 456-460.

[34] E. Tsai, J. M. Richardson, E. Korblova, M. Nakata, D. Chen, Y. Shen, R. Shao, N. A. Clark, D. M. Walba, Angew. Chem. Int. Ed. 2013, 52, 5254-5257.

[35] T. Ueda, S. Masuko, F. Araoka, K. Ishikawa, H. Takezoe, Angew. Chem. Int. Ed. 2013, 52, 6863-6866.

[36] N. Gimeno, M. B. Ros, Handbook of Liquid Crystals Vol.4, Wiley-VCH, Weinheim, 2014.

[37] E. Gorecka, N. Vaupotic, D. Pociecha, Handbook of Liquid Crystals Vol.4, Wiley-VCH, Weinheim, 2014.

[38] L. Li, M. Salamonczyk, A. Jákli, T. Hegmann, Small 2016, 12, 3944-3955.

[39] L. Li, S. Shadpour, C. Zhu, A. Jákli, T. Hegmann, Nat. Commun. 2018, 9, 1-8.

[40] S. Shadpour, A. Nemati, N. J. Boyd, L. Li, M. E. Prevot, S. L. Wakerlin, J. P. Vanegas, M. Salamonczyk, E. Hegmann, C. Zhu, M. R. Wilson, A. I. Jákli, T. Hegmann,, Mater. Horiz. 2019, 6, 959-968.

[41] D. Chen, C. Zhu, H. Wang, J. E. MacLennan, M. A. Glaser, E. Korblova, D. M. Walba, J. A. Rego, E. A. Soto-Bustamante, N. A. Clark, Soft Matter 2013, 9, 462-471.

[42] C. Zhang, N. Diorio, O. D. Lavrentovich, A. Jákli, Nat. Commun. 2014, 5, 1-6.

[43] R. A. Callahan, D. C. Coffey, D. Chen, N. A. Clark, G. Rumbles, D. M. Walba, ACS Appl. Mater. Interfaces 2014, 6, 4823-4830.

[44] K. Kim, H. Kim, S. Y. Jo, F. Araoka, D. K. Yoon, S. W. Choi, ACS Appl. Mater. Interfaces 2015, 7, 22686-22691.

[45] Y. Shi, Z. Sun, R. Chen, C. Zhu, R. K. Shoemaker, E. Tsai, D. M. Walba, M. A. Glaser, J. E. Maclennan, D. Chen, N. A. Clark, J. Phys. Chem. B 2017, 121, 6944-6950.

[46] D. Chen, J. E. MacLennan, R. Shao, D. K. Yoon, H. Wang, E. Korblova, D. M. Walba, M. A. Glaser, N. A. Clark, J. Am. Chem. Soc. 2011, 133, 12656-12663.

F. Araoka, G. Sugiyama, K. Ishikawa, H. Takezoe, Adv. Funct. Mater. 2013, 23, 2701-2707.

D. M. Walba, E. Körblova, R. Shao, J. E. Maclennan, D. R. Link, M. A. Glaser, N. A. Clark, Science 2000, 288, 21812184.

[49] D. M. Walba, L. Eshdat, E. Körblova, R. K. Shoemaker, Cryst. Growth Des. 2005, 5, 2091-2099. 
[50] J. Etxebarria, M. B. Ros, J. Mater. Chem. 2008, 18, 29192926.

[51] C. Zang, N. Diorio, A. Jakli, Handbook of Liquid Crystals Vol.4, Wiley-VCH, Weinheim, 2014.

[52] S. C. Lin, T. F. Lin, R. M. Ho, C. Y. Chang, C. S. Hsu, Adv. Funct. Mater. 2008, 18, 3386-3394.

[53] S. C. Lin, R. M. Ho, C. Y. Chang, C. S. Hsu, Chem. Eur. J. 2012, 18, 9091-9098.

[54] M. Yoshio, R. Konishi, T. Sakamoto, T. Kato, New J. Chem. 2013, 37, 143-147.

[55] A. Zep, M. Salamonczyk, N. Vaupotic, D. Pociecha, E. Gorecka, Chem. Commun. 2013, 49, 3119-3121.

[56] M. F. Rizkiana, R. Balamurugan, J. H. Liu, New J. Chem. 2015, 39, 6068-6075.

[57] J. Matraszek, N. Topnani, N. Vaupotič, H. Takezoe, J. Mieczkowski, D. Pociecha, E. Gorecka, Angew. Chem. Int. Ed. 2016, 55, 3468-3472.

[58] M. Castillo-Vallés, A. Martínez-Bueno, R. Giménez, T. Sierra, M. B. Ros, J. Mater. Chem. C 2019, 7, 14454-14470.

[59] B. Donnio, S. Buathong, I. Bury, D. Guillon, Chem. Soc. Rev. 2007, 36, 1495-1513.

[60] C. F. C. Fitié, I. Tomatsu, D. Byelov, W. H. De Jeu, R. P. Sijbesma, Chem. Mater. 2008, 2394-2404.

[61] H. P. C. Van Kuringen, G. M. Eikelboom, I. K. Shishmanova, D. J. Broer, A. P. H. J. Schenning, Adv. Funct. Mater. 2014, 24, 5045-5051.

[62] H. J. Sun, S. Zhang, V. Percec, Chem. Soc. Rev. 2015, 44, 3900-3923.

[63] A. Concellón, M. Marcos, P. Romero, J. L. Serrano, R. Termine, A. Golemme, Angew. Chem. Int. Ed. 2017, 56, 1259-1263.

[64] A. Concellón, T. Liang, A. P. H. J. Schenning, J. L. Serrano, P. Romero, M. Marcos, J. Mater. Chem. C 2018, 6, 10001007.

[65] A. Concellon, R. Termine, A. Golemme, P. Romero, M Marcos, L. José Serrano, J. Mater. Chem. C 2019, DOI 10.1039/c8tc06142d.

[66] Y. Zhang, U. Baumeister, C. Tschierske, M. J. O'Callaghan, C. Walker, Chem. Mater. 2010, 22, 2869-2884.

[67] J. Vergara, N. Gimeno, M. Cano, J. Barberá, P. Romero, J. L. Serrano, M. B. Ros, Chem. Mater. 2011, 23, 4931-4940.

[68] A. Concellón, S. Hernández-Ainsa, J. Barberá, P. Romero, J. L. Serrano, M. Marcos, RSC Adv. 2018, 8, 37700-37706.

[69] F. Gröhn, Macromol. Chem. Phys. 2008, 209, 2291-2301.
[70] S. Hernández-Ainsa, J. Barberá, M. Marcos, J. L. Serrano, Soft Matter 2011, 7, 2560-2568.

[71] E. Fedeli, S. Hernández-Aínsa, A. Lancelot, R. GonzálezPastor, P. Calvo, T. Sierra, J. L. Serrano, Soft Matter 2015, 11, 6009-6017.

[72] M. Cano, A. Sanchez-Ferrer, J. L. Serrano, N. Gimeno, M. B. Ros, Angew. Chem. Int. Ed. 2014, 53, 13449-13453.

[73] R. Oda, I. Huc, M. Schmutz, S. J. Candau, F. C. MacKintosh, Nature 1999, 399, 566-569.

[74] J. V. Selinger, M. S. Spector, J. M. Schnur, J. Phys. Chem. B 2001, 105, 7157-7169.

[75] L. Ziserman, H. Y. Lee, S. R. Raghavan, A. Mor, D. Danino, J. Am. Chem. Soc. 2011, 133, 2511-2517.

[76] C. Valéry, F. Artzner, M. Paternostre, Soft Matter 2011, 7, 9583-9594.

[77] J. Adamcik, R. Mezzenga, Macromolecules 2012, 45, 11371150.

[78] R. Mezzenga, J. Adamcik, Angew. Chemie Int. Ed. 2018, 57, 8370-8382.

[79] R. Martín-Rapún, M. Marcos, A. Omenat, J. Barberá, P. Romero, J. L. Serrano, J. Am. Chem. Soc. 2005, 127, 73977403.

[80] J. Thisayukta, Y. Nakayama, S. Kawauchi, H. Takezoe, J. Watanabe, J. Am. Chem. Soc. 2000, 122, 7441-7448.

[81] M. W. Schröder, S. Diele, G. Pelzl, W. Weissflog, ChemPhysChem 2004, 5, 99-103.

[82] Y. Yamamura, R. Tsuchiya, S. Fujimura, M. Hishida, K. Saito, J. Phys. Chem. B 2017, 121, 1438-1447.

[83] E. T. Pashuck, S. I. Stupp, J. Am. Chem. Soc. 2010, 132, 8819-8821.

[84] L. Ziserman, A. Mor, D. Harries, D. Danino, Phys. Rev. Lett. 2011, 106, 10-13.

[85] J. Adamcik, V. Castelletto, S. Bolisetty, I. W. Hamley, R. Mezzenga, Angew. Chem. Int. Ed. 2011, 50, 5495-5498.

[86] T. Shimizu, M. Masuda, H. Minamikawa, Chem. Rev. 2005 $105,1401-1443$

[87] H. Cui, T. Muraoka, A. G. Cheetham, S. I. Stupp, Nano Lett. 2009, 9, 945-951. 